\title{
Erratum: Polarization measurement of dielectronic recombination transitions in highly charged krypton ions [Phys. Rev. A 92, 042702 (2015)]
}

Chintan Shah, Holger Jörg, Sven Bernitt, Stepan Dobrodey, René Steinbrügge, Christian Beilmann, Pedro Amaro, Zhimin Hu, Sebastian Weber, Stephan Fritzsche, Andrey Surzhykov, José R. Crespo López-Urrutia, and Stanislav Tashenov (Received 3 August 2016; published 15 August 2016)

DOI: 10.1103/PhysRevA.94.029905

In the original paper, Eq. (5) should be corrected to

$$
\mathcal{A}_{k}=\sqrt{2 J_{d}+1} \sum_{M_{d}=-J_{d}}^{J_{d}}(-1)^{J_{d}-M_{d}}\left\langle J_{d} M_{d}, J_{d}-M_{d} \mid k 0\right\rangle \frac{\sigma_{M_{d}}}{\sum_{M_{d}^{\prime}} \sigma_{M_{d}^{\prime}}} .
$$

Additionally, Eq. (10) should be replaced with

$$
P(\theta)=\frac{\sum_{k=2,4, \ldots} 2 \sqrt{\frac{(k-2) !}{(k+2) !}} \mathcal{A}_{k} g_{k} P_{k}^{(2)}(\cos \theta)}{1+\sum_{k=2,4, \ldots} \mathcal{A}_{k} f_{k} P_{k}(\cos \theta)} .
$$

The corrected forms of Eqs. (5) and (10) were already employed in our theoretical calculations. Therefore, these corrections in particular do not affect any other section of the paper, leaving unchanged our overall discussion and conclusions. 